\title{
Quantum Entangled Frequencies and Coherence in Bioenergetic Systems:
}

\section{Information Field Processes related to the Concepts of Akasha and Prana}

\author{
Marcus Schmieke ${ }^{1 *}$ \\ ${ }^{1}$ Head of scientific research, Institute of Existential Consciousness Research (ECR), Berlin, Germany \\ ${ }^{1}$ Adjunct Professor, Dev Sanskriti University, Haridwar, India \\ https://doi.org/10.36018/dsiij.v18i.226
}

\begin{abstract}
Holistic Health can be understood as the coherence of the human being as a whole including his organism, mind, and relationship to his environment. Quantum entanglement of subsystems on all levels of the human being is responsible for the wholeness of the living entity. The sum total of entanglements is defined here as an individual Information Field which corresponds to the ancient vedic concept of akasha. Entangled frequencies might be able to resonate with and influence human bioenergetic processes and systems with the aim of creating coherence of the bioenergetic system, both within the individual and between the individual and the surrounding fields. Here a bioenergetic process is proposed that uses entangled frequencies to increase the coherence of living systems selected through biofeedback of a Quantum Noise Generator, which seems to be correlated to the ancient vedic concept of prana. It has been tested in a controlled observation study for its effect on the sense of coherence of 3545 participants. The promising results of this study are additionally presented here.
\end{abstract}

Keywords. Bioenergetic; Entangled Frequencies; Quantum Entanglement; Teleportation; Generalized Quantum Theory; Information Field; Coherence; Prana; Akasha; Chi; Coherence

\section{The Bioenergetic Quantum Nature of Biological Systems}

Quantum Influences on Bioenergetic and Bioelectric Systems

The term "bioenergetic" in this regard refers to the influence of quantum processes on the bioelectric and electromagnetic systems, processes, and fields of the body (Popp et al. 1984 (1), Popp, Y. Yan 2002 (2), Popp Chang 2002 (3), Jacobson 1989 (4)). Beginning with photons initiating biochemical processes in the body-and progressing to the electric fields of cells and organs, heart, brain at all levels of complexity - the physical and biochemical systems of the body are accompanied (or even controlled) by electromagnetic fields and electrical currents. These electromagnetic and electrical processes are characterized on many levels by holistic quantum phenomena (Popp et al 1984 (5), Francisco Di Biase 2017 (6)). The quantum interactions of these bioelectric and bioelectromagnetic structures and processes unite to form the bioenergetic field of a human being. It includes not only the body, but its interactions with the mind, the deeper psyche, and consciousness, and can be related to ancient concepts like Chi and Prana that are foundational to traditional Chinese Medicine 
(TCM) and the Yoga traditions of India ${ }^{1}$ (K. J. Hintz, G.L. Yount, I. Kadar, G. Schwartz, R. Hammerschlag, S. Lin, 2003 (7)), also (Shyang Chang2012 (8)), (Chaing et al. 2014 (9)).

For thousands of years, terms like Chi and Pranareferring to the bioenergetic field of the body and to the term "life energy" — were exclusively the territory of Eastern philosophers and metaphysicians.

\section{The Information Field}

For the purposes of this discussion, we refer to the quantum processes of body, mind, and the deeper psyche as a person's individual Information Field. It consists of the quantum information contained in the sum of generalized quantum entanglements (Primas 2003 (10)), (Walach, Lucadau 2007 (11)), (Atmanspacher, Römer, Wallach 2002 (12)) both in the human being and between the human being and the environment. The subset of an individual's Information Field is recognized by the $\mathrm{NIH}$ (National Institutes of Health) as the "bioenergetic field”. In the ancient Vedic texts it corresponds to the concept of akasha as the central element of the Sankhya-philosophy connecting the realms of gross and subtle matter. The bioenergetic field communicates to the biological body via dynamic quantum processes.

A general concept of an Information Field has been developed by scientists of various disciplines

\footnotetext{
1"The word bioenergy has been used to encompass a set of terms that may or may not belong in the same physical category. In western cultures, these terms include electromagnetic and quantum fields when applied to energy medicine. The term subtle energy is also sometimes used, as are vital force and L-energy (for life energy). In eastern cultures, energy terms include qi or chi (China), ki (Japan), as well as prana (India) and mana (Hawaii I Philippines)" (K. J. Hintz, G.L. Yount, I. Kadar, G. Schwartz, R. Hammerschlag, S. Lin, 2003 (7)) also, (Shyang Chang 2012 (8)), (Chaing et al. 2014 (9)).
}

(Zeilinger 1998 (13), Wallach 2005 (14), AharonovBohm 1959 (15), Bouwmeester, Zeilinger et al. 1997 (16), Atmanspacher, Walach 2002 (17), Tiller 1993 (18), Sheldrake 2006 (19), Laszlo 2004 (20))². A similar concept regarding cellular biology, with clear definitions of global and individual Information Fields, is presented by William B. Miller (Miller 2017 (21)):

It has been previously advanced that all living entities assess information beyond their immediate environment and exist as part of a system of Pervasive Information Fields (PIFs) (Miller, 2016 (22)). These fields of information are best modeled after the concept introduced by Lloyd (2002) (23) as selforganizing, universal, and scale-free informational sets that can be appropriately applied to biological organisms. Within this frame, each living organism has its own unique Information Field within space-time as an attachment to outward information space. This is composed by its physical environment and by the overlap of all the individualized Information Fields of each of the varied constituents with which it can communicate, both near and far.

\footnotetext{
2 Anton Zeilinger has established (in his publications and experiments) that quantum information is the most fundamental physical reality and that it is the foundation of all physical processes. Further, he uses the entanglement of quantum information as evidence and support for quantum teleportation (Bouwmeester, Zeilinger et al. 1997 (16)). Similarly, Harald Walach-in the frame of Weak Quantum Theory (WQT) (Atmanspacher, Walach 2002 (17))—developed a theory applying a generalized version of quantum theory to establish generalized quantum entanglement as a basis of generalized quantum teleportation. In the context of WQT, the underlying generalized quantum information is the Information Field to which we are referring. In a 1959 publication, Aharonov and Bohm posit an electromagnetic quantum field which, although undetectable, affects charged particles. William Tiller also refers to a quantum Information Field undetectably hidden in the Dirac sea of negative energy (Tiller 1993 (18)). The British biologist Rupert Sheldrake popularized this concept of the Information Field (in a biological and anthropological context) as the morphic or morphogenetic field (Sheldrake 2006 (19)). Also, Erwin Laszlo developed a theory of the Information Field as the Akashic Field (Laszlo 2004 (20)). As the result of scientific investigation and speculation, the Information Field has become of increasing interest to interdisciplinary scientists as a valid alternative to a purely materialistic worldview.
} 
The Addition of Wholeness to Fully Local and Analytic Models

Holistic quantum models of an organism represent an evolutionary step to understanding being, and only recently is it becoming understood by the analytic terminology of current biology. Biology and medicine tend to consider the human being as consisting of many systems, such as mind and body, organs, functional systems, cells, cell organelles, biomolecules, etc. This analysis tends to overlook the wholeness of the human being, treating the body as analytically fragmented into hierarchical systems. Lee Smolin and Roberto Unger in their book The Singular Universe and the Reality of Time (Unger, Roberto Mangabeira; Smolin, Lee 2015 (24)) state that biology and medicine are still using concepts of Aristotelian physics, outdated for centuries since Isaac Newton,whose ideas have been extended by Einstein's demonstration of the common nature of energy and mass as two forms of the same essence.

The discipline of biophysics started a few decades ago to apply these modern concepts of physics, especially quantum theory, to biology. (Schrödinger 1944 (25), S. Davydov 1982 (26), Graham R. Fleminga, Gregory D. Scholes, Yuan-Chung Cheng 2011 (27)) As a result, terms like "bioenergetic fields" have achieved a new scientific meaning, reestablishing anevolving awareness of the wholeness and interactions of the apparently separated objects of the body.

A new proposal in this paper is to define the bioenergetic field of a living entity as the bodyrelated component of its individual Information Field. The individual Information Field is proposed to be defined as the complete information contained in the holistic sum of its generalized quantum entanglements (Hintergerberet al, 2012 (28)).
(Atmanspacher, Römer, Wallach 2002 (29); Wallach 2005 (30)) The bioenergetic field communicates to the electrical and electromagnetic levels of the body by related quantum processes. Therefore the electromagnetic level is the focus of Information Field controlled frequency applications. This electromagnetic level functions as the link integrating the bioenergetics of the body with the essence of the Information Field.

Although biology and medicine apply advanced models of physics, each still uses Aristotelian logic, which is linear and dualistic. These are most valuable in clinical and emergency interventions, such as repairing a broken leg. Unfortunately, this twovalued dualistic approach sacrifices a sense of the wholeness of nature which is essential for optimum health.

More complex logical systems and concepts of nature need to be applied to get a deeper understanding of life, meaning, and consciousness, which are crucial for both individual and community health and wellbeing (Schmieke, 2021) (31).

Quantum Effects of Bioelectric Fields and Wholeness

A quantum object is characterized by the quality of wholeness and inseparability, which means that it can't be divided into parts without losing the important phase relationship information between the resulting parts. In the fragmented system, the lost quantum information reappears by nonlocal and acausal effects of quantum entanglement. This appears in classical quantum theory applied to the microscopic level of the body, but also in macroscopic biophysical structures of the body, especially in the nervous system and the immune system (Stuart Hameroff, Roger Penrose 1996 (32)) 
(Lin et al. 2015 (33)). The separation of body, mind, and soul-which is generally a basis of scientific analytic approaches - results in the appearance of unexpected nonlocal and acausal phenomena in the remaining physiological system (Martin, Carminati et al. 1997 (34)). It also results in the duality of the Copenhagen interpretation of quantum-mechanicsappearing between the Unitarian development of the wave function between measurements and its spontaneous reduction-strangely suggesting a nonphysical observer and contradicting the Schrödinger equation itself (Lee Smolin, 2017 (35)).

The loss of wholeness is the price paid bythe purely quantitative description of a two-valued logicsystem.

\section{Generalized Quantum Theory and Quantum Entanglement in macroscopic Systems}

As predicted by the generalized quantum theory of Atmanspacher, Römer, and Wallach (Atmanspacher et al. 2002 (36)), wholeness reappears in phenomena of macroscopic quantum entanglements. This also is evident on many levels of biophysical structures where coherence appears as an indication of the hidden wholeness of the organism. Bioenergetic properties and systems are a compensation for the quantum wholeness sacrificed by modern scientific models. But combining the quantum concepts of coherence, entanglement, and non-local interactions with traditional terminology of bioenergetic systems — such as the meridians and the chakras-is leading to convergence. Large amounts of research have been done on the scientific meaning and correlation of those terms. But the exploration has only begun! Due to newly available tools of research and observation, we have begun to mine a deeper understanding of health and well-being through holistic quantum effects and information (Brizhik et al. 2018 (37), Yang and Han 2015 (38)).

\section{Definition of Bioenergy in Terms of Coherence}

On the basis of these assumptions the bioenergetic state of an organism is proposed in this paper to be proportional to

- $\quad$ the degree of coherence (Bajpai 2003 (39)) of its bioelectrical field and

- the quantity of its coherent energy

Coherent energy is the potential to achieve the purpose of a specific expression of life (especially its meaning) within its environment.

Parallel to this definition, the bioenergy of a living organism is proportional to the sum total of its positive possibilities, which is expressed in coherent and focused information contained in the system.

Coherence, according to Marco Bischof, appears in many measurable expressions of life in the organism and also on a mental and psychological level (Marco Bischof 1996 (40)). The highest degree of coherence is achieved if body, mind, and soul are in one coherent state, which is traditionally referred to as "the state of enlightenment."

Modern science has researched enlightenment states using

- EEG (Electroencephalography) neuroimaging and

- heart-brain coherence measurements (coherent correlations of HRV (Heart Rate Variability) (Khandoker et al. 2008 (41)).

\section{Bioenergy as a Flow of Information} The bioenergetic field is the Information Field of a human being, connecting to the human being as a whole and to the human body. This is especially true of the holistic, coherent, and quantum aspects ${ }^{3}$ of the

\footnotetext{
${ }^{3}$ Broadly speaking, quantum mechanics incorporates four classes of phenomena for which classical physics cannot account: quantization of certain physical properties, quantum entanglement, principle of uncertainty, wave-particle duality. (Wikipedia)
} 
body's electromagnetic and electrical fields. The traditional life energy concepts of Prana and Chi are not related to physical energy but rather to dynamic flows of information (Michael von Brück,2014 (42)). Weizsäcker, as the assistant of Werner Heisenberg, was one of the best thinkers regarding quantum philosophy. He compared the terms life energy, prana, or bioenergy to the probability amplitude of the wave function, believing it to be a quantified expression of where time leads evolution (Weizsäcker, Gopi Krishna, 2010 (43)). Therefore it is quantum information on the least concrete level of physical expression. As the Information Field of the human system, this quantum information connects body, mind, and soul to restore the wholeness of the human being. It restores what is lost by internal psychological or external environmental influences. Since the Information Field is the sum total of generalized quantum entanglements connecting a human being to the environment, it recognizes the integration of parts on the level of quantum information (Primas 2003 (44)). The Information Field is the global frame in which all local systems of a more concrete or physical nature are embedded.

\section{Effects of Frequency Applications by Generalized} Quantum Entanglement

According to the Generalized Quantum Theory or Weak Quantum Theory (WQT) of Atmanspacher, Römer, and Wallach, global systems are quantum entangled with their parts, and the parts are quantum entangled with each other if the global and local systems possess complementary properties which are non-commutable. In the same way, quantum mechanics posits that the location of a particle and its momentum are non-commutable observables(Ochoa, et al. 2018 (45)). This means that sequence in which they are measured in a particular system is important. (Atmanspacher, Römer, Wallach, 2002 (46); Wallach 2005 (47) $)^{4}$ This is the minimum set of requirements for a system in order to develop properties like generalized quantum entanglement.

Here a bioenergetic process is proposed from the perspective of WQT, which applies electromagnetic frequencies for example by microcurrent to the human body with low amplitudes which are selected by an open quantum process used as a random event generator (REG). The REG is expected to show effects of quantum entanglements with the meaning of the frequencies (content of the Information Field), the bioenergetic field and bioelectrical resonances of the frequencies. This influences the physical processes in the bioelectric field ${ }^{5}$ in addition to the direct effect of the frequencies on the bioelectrical level.

The purpose is always to create coherence, thus promoting general wellbeing and holistic health. This is achieved through bioenergetic frequencies of low amplitude (microcurrent) and global application (mostly through wrist electrodes to cover all the

\footnotetext{
${ }^{4}$ Walach comprehends the Weak Quantum Theory (WQT) in a concise form:"WQT predicts entanglement between elements of a system if two variables or observables are complementary: one describing a global and one the local aspects of the system. Entanglement then ensues between those local elements of a system that are complementary to the global description or observable of that system" (Walach2005 p. 1 (107)).

Atmanspacher, Römerand Walach give a simple set of conditions a system has to fulfil, so that is exhibits generalized quantum entanglements among its subsystems: "In general, incompatible observables do not have simultaneous definite values. Although the generalized weak quantum theory as defined by axiomsI-VI is considerably weaker than ordinary quantum theory, they share the following two characteristic features.

- Incompatibility and complementarity arise due to the non-commutativity of the multiplication of observables.

- Holistic correlations and entanglement arise if for a composite system observables pertaining to the whole system are incompatible with observables of its parts" (2002Atmanspacher, Römer, Walach, 2002 p. 14 (35))
}

${ }^{5}$ The WQT quantum entanglement effect will be discussed in detail in the second chapter of this article. 
meridians) to influence the Information Field of the body and develop towards greater coherence towards the intended focus.

As a consequence, bioenergetic frequencies can benefit the coherence of the bioelectrical field of the human being through generalized quantum entanglement. Additionally, the frequencymodulated microcurrent can manifest its direct effect on the bioelectrical level of the body and the cells. In the third chapter of this paper, a controlled trial is documented using frequencies to promote coherence.

Model of interaction between the material body and Information Field

This diagram illustrates the effect of frequencies, putting them into a meaningful context of physics, biology, and Information Field theory. In its symmetrical structure, matter and information space are mirror images of each other. They are connected by the mirror axis of the quantum process, through which the exchange between matter and the Information Field takes place. Quantum processes are non-deterministic physical random processes. As an alternative to the combination of deterministic natural laws and random processes, they permit information flow from the Information Field towards the matter.

Using acausal and non-local effects, quantum processes connect remote spatial areas. The matter is represented by the molecular body and electromagnetic fields. The electromagnetic body consists of the electrical level of the cell and tissue and the electromagnetic interactions, fields, and radiation. These extend to the ultra-weak cell radiation, the so-called biophotons (Popp et al. 1984 (48)). If a reduction of the wave function takes place on the quantum level of these electromagnetic processes, all quantum entanglements associated with it (even in a generalized form) and related to the respective process flow into it. Therefore, the diagram indicates the level of quantum processes and the area of locally/not locally effective quantum entanglements in which the hidden relationships of the fragmented material reality are contained.

The material reality is a classical approximation, attained by neglecting quantum physical phase relationships and aspects of wholeness. Thus, individual objects come to the fore of consciousness. The lost wholeness and relationships reappear as quantum entanglements.

The sum total of the information contained in a person's quantum entanglements (both internal and external) is what in this paper is defined as his or her individual Information Field. That component related to the body is the person's bioenergetic field. It connects the body with the mind and soul and therefore, may be regarded as the essence of life. Mind and soul in this context refer to the conscious (mind) and unconscious (soul) aspects of the human psyche, together forming the wholeness of internal experiences and reality. In this way, the Information Field is not an additional ontological field, but the necessary complement to the classical approach to objects existing separately from each other (Görnitz 2012 (49)). It is not a speculative addition to reality, but the necessary complement to the classical approximation of reality through hidden quantum entanglements. However, such a classical approximation has to be assumed in each model; because, in quantum physics, a part of reality has to be described classically in order to obtain measurable results. 


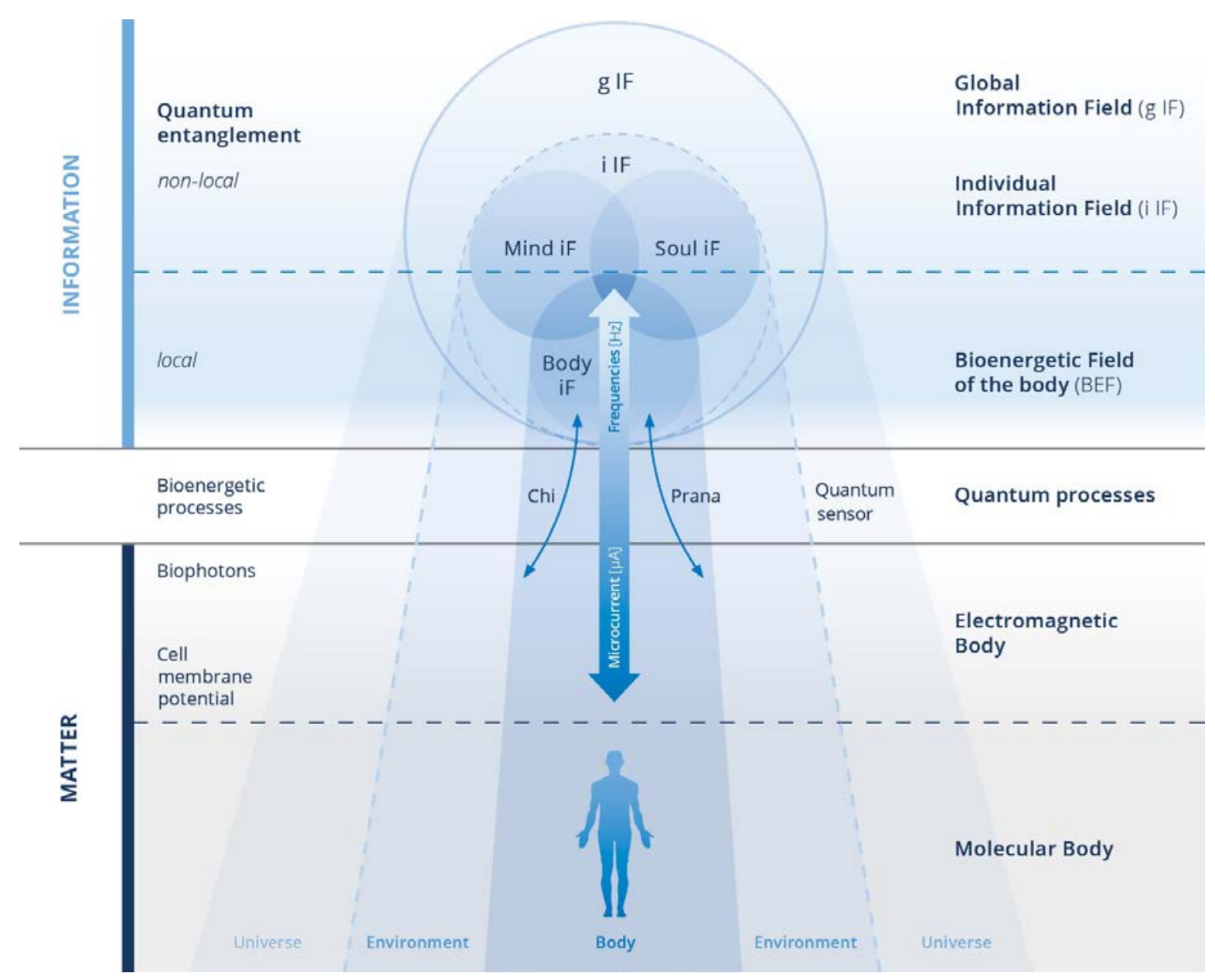

Figure 1.Matter and Information as two sides of reality interacting through quantum processes

The Information Field, consisting of the sum of the hidden generalized and strong ${ }^{6}$ quantum entanglements, can be divided into three areas:

1. The global Information Field refers to the entire universe and the relationships of its individual subsystems and living beings to each other.

2. The individual Information Field contains the information of all quantum entanglements related to an individual living being. Within the individual Information Field, there are three areas relating to the trinity of a) body, b) mind (conscious), and c) soul (unconscious).

3. The bio-energetic field is the area of the Information Field related to the body and connects body, mind, and soul.

\footnotetext{
${ }^{6}$ Strong quantum entanglement fulfills all the entanglement conditions of quantum theory whereas a weak or generalized entanglement just fulfills the weaker conditions of WQT.
}

The Information Field controlled frequency applications of the proposed bioelectric process emanate in both directions from the middle of the diagram. The process is shown in the middle because the physical noise generator (REG or quantum sensor) in the center of such a process determines the current application frequency on the basis of physical quantum processes. This frequency is then modulated on the microcurrent. The low microcurrent frequencies up to approx. $130 \mathrm{~Hz}$ (50) act at the level of cell electricity and are intended to increase the cell membrane potential and thus the cell energy. Higher frequencies mainly harmonize the bioenergetic field in a sequence selected by the Information Field, supporting the wholeness of body, mind, and soul (coherence). As claimed by Miller 
(William Miller 2016 (50)), the coherence of the Information Field of the cell is closely related to the integrity and properties of the cell membrane. An example is the cell membrane potential:

"The cellular boundary delimits the informational field, shielding it from some distortions or deformations caused by external environmental variables and adjacent cellular field effects. The cell membrane creates the environment in which the integrity of the Information Field can be protected and coherently projected."

Again, this component of the Information Field is called the Bioenergetic Field of the cell.

The importance of frequencies in the quantum nature of life is expressed by Miller (William Miller 2016 (52)):

"Life has been pictured in that frame as a farfrom-equilibrium coherent photon field in a range of frequencies. The differing components of the organism, each with their unique characteristics, nevertheless synchronize together through quantum coherent fields.”

Frequencies can thus be described as a language by which the global and individual Information Fields communicate with the electromagnetic and bioenergetic field of the body. Miller (William Miller 2016 (53)) supports this concept theoretically by relating energy transfer and Information to frequency and amplitude:

"However, as energy transfer is an oscillating function of frequency and amplitude, there must be zones of coherence (amplification or resonance) or decoherence that occur across gradients within any boundary condition.”

Further, Miller identifies energy transfer with information and the boundary of the cell (cell membrane) as the resonance space which transforms energy into information and vice versa:

"Since information is energy transfer, information becomes a gradient function subject to harmonics and resonances that instantiates or promotes a spectrum of awareness of status. Therefore, each cellular unit is a coherent and discrete cognitive entity in which information becomes another form of resonant energy both within and without the cell. Energy becomes information within the bounded resonating chamber of the cell achieving the coherence necessary to become information to both sender and receiver."

The dynamic mutual transfer of energy and information as an essential life process is subject to frequencies, harmonics, and resonances. These frequencies generated by quantum processes within the bioenergetic process connect the global Information Field to the bioenergetic field of the cell and the body to unite body, mind, and soul by generalized quantum entanglement. In this process, each cell is a cognitive unit with its own Information Field(the bioenergetic field of the cell) communicating through electromagnetic frequencies with its environment, other cells, and the underlying Information Field.

In this model, frequencies take on a similar function to that of bioenergetic processes, which in traditional cultures are called life energy, chi, or prana. They convey the influence of the three Information Fields to the body and make that influence perceptible.

\section{Coherent Frequency Patterns as an Informational} Basis of Life

The fundamental effect of frequencies as an informational basis of life has been suggested by a series of research papers by Geesink and Meijer 2017 (54). Through the analysis of more than 500 
studies on the effects of frequencies on living systems, the authors found a scale-invariant pattern of frequencies in the range from $0,1 \mathrm{~Hz}$ to beyond $500 \mathrm{THz}$ that affects living systems in a positive way by promoting their degree of coherence (Gessink, Meijer 2016 (55)). The same pattern of coherence promoting frequencies is found in the infrared spectrum of pure water (Geesink, Jerman, Meijer 2020 (56)) and of clay (Geesink, Jerman, Meijer 2020 (57)) which according to the authors may serve as replicators of an underlying frequency pattern of the quantum vacuum. The authors express their view in the abstract of a paper, published in 2020 (58):

"Meta-analyses of current biophysical literature revealed the effects of similar EMF frequency patterns in a wide range of animate and non-animate systems. This provided a novel conceptual bridge between living and non-living systems, being of relevance for the areas of biophysics, brain research, as well as for mechanisms of biological evolution. As to the latter aspect, the potential role of coherently structured water is treated in relation to the generation of a primordial biofield,that is seen as instrumental in a partially guided creation of first life. We hold, in general, that nature is guided by a discrete pattern of harmonic, solitonic, waves, likely originating from quantum vacuum fluctuations derived from an immanent zero-point energy (ZPE)/ superfluid quantum space. We propose, therefore, that the pro-life EM frequency bands, identified in our earlier studies may literally act in concert as "tonal octave-based symphony" to provide living systems, including the brain, with information embedded in such harmonic-like resonance patterns”.
In a paper "Water, the cradle of life" (Geesink, Jerman, Meijer 2020 (59)) the authors draw a systematic conclusion about the coherent frequencies in water as the basis for cellular life processes. In this section, the authors draw the conclusion, that coherent frequencies promote quantum entanglement (Fröhlich 1968 (60)) on the intra- and extracellular levels and in this way support the development of larger coherent domains (Bischof, Del Giudice 2013 (61)). By their positive influence on the degree of entanglement on the cellular level, coherent frequencies can also be instrumental to harmonize the Information Field of the cells, which is defined by the sum total of the quantum entanglements of the cells, internally and externally. The degree of the cell's internal and external quantum entanglement is an indicator for their ability to communicate with other cells and the degree of internal communication of its parts and components like the DNA, mitochondria, or cell-organelles. As Geesink and Meijer's research and conclusions are based on more than 500 studies of the biological and medical effects of frequencies, they are a strong basis for further research on the effect of frequencies on cells, organisms and their underlying Information Field. Meijer and Geesink understand the coherent frequency pattern to be resonance frequencies from the underlying quantum vacuum, which they consider to be closely related to the Information Field in a Bohmian sense (62). Coherent frequencies therefore may serve to strengthen the resonance of coherent domains of living organisms to the underlying Information Field/quantum vacuum. Meijer and Geesinks research supports the assumption, that the octave principle of harmonics may be applied to coherent frequencies also (Meijer, Geesink 2018 (63)). This means that a coherent frequency is obtained by multiplying a coherent frequency with a multiple of two $\left(2^{\mathrm{n}}\right)$. In the second 
part of this paper 214 of these frequencies from 0,4 $\mathrm{Hz}$ to $1 \mathrm{MHz}$ are tested in a controlled trial for their influence on the sense of coherence of healthy humans.

\section{The Information Field in Physics and Biology}

Information Influences on Physical Random Processes

Two Decades of PEAR(Princeton Engineering Anomalies Research Laboratory)(Robert G. Jahn, Brenda JDunne, 1999), (64) an anthology of selected publications,collects the research papers of Brenda Dunne and Robert Jahn on consciousness effects on random physical processes at PEAR Lab at the University of Princeton (Jahn, Dunne, 2005 (65)). ${ }^{7}$ The result of this research concluded in meta-studies clearly indicates that physical random processes based on open quantum processes can reflect intentions and other mental qualities (Radin, Nelson, 1989 (66)). Random physical processes can also be influenced by related emotions by the intention of the experimenter (Blasband 2000 (67)).Roger Nelson, a renowned psychologist as the director of the physical PEAR Lab at Princeton conducted his own research in the Global Consciousness Project, measuring the effect of global happenings on the frequency distribution of a large network of up to 80 physical noise generators all over the world (Roger Nelson, 2008 (68)). Nelson correlated the temporal development of the deviation of the total noise data from its expected value with selected significant events, such as the terrorist attacks of September 11 or the fatal accident of Lady Diana. Nelson concluded that events that make a strong emotional impact on the collective consciousness are statistically significantly correlated with a strong

\footnotetext{
${ }^{7}$ Current publication from the Princeton University Library and Press with relevance to Consciousness, creativity, and self can be found on its website.
}

deviation of the cumulative noise data from the expectation value. He published his research conducted over 20 years in a book called "Connected: The Emergence of Global Consciousness”, (Roger D. Nelson, Dean Radin 2019 (69)), postulating the existence of a global mind of humanity, which corresponds to a global Information Field and modulates physical random processes. The work of Nelson and Radin indicates that the signals of physical noise generators could carry information based on the intention of the users and a potentially existing global Information Field (an emerging field called Global Consciousness by Roger Nelson) ${ }^{8}$.This global Information Field is the global counterpart to and interacts with the individual Information Field of the human being. The influence of conscious intention was also confirmed by the work of the German scientist Helmut Schmidt, whose experiments (partially together with Henry Stapp (70) from Harvard University) are published in numerous scientific articles (Helmut Schmidt, 1971-1997 (71)). From all this research it can be concluded that the global Information Field and the individual Information Field through generalized quantum entanglement can transfer information between the individual and the collective on the electrical signals of physical noise generators based on quantum processes. In the proposed bioenergetic process this information is used to pick frequencies for modulating the microcurrent. Frequencies in this way act as a language to transfer holistic quantum information (coherent information from the global Information Field) to the individual's Information Field to

\footnotetext{
${ }^{8}$ As suggested by Beenakker and Schönenberger 2003 (88): "The noise is the signal" was a saying of Rolf Landauer, one of the founding fathers of mesoscopic physics. What he meant is that fluctuations in time of a measurement can be a source of information that is not present in the time-averaged value. A physicist may actually delight in "noise".
} 
increase its coherence and connectedness to the global Information Field.

Frequencies-modulated electrical fields and currents that enforce coherent states of neuronal activities have been experimentally demonstrated (Frohlich, F. \& McCormick, D.A. 2010 (72)) (Singer 2011 (73)). These coherent states of neuronal firing produce an electrical field that has been identified experimentally as the seat of consciousness (McFadden 2002 (74)). In return, these electrical fields influence the neuronal activities and create pressure on them to develop in a certain direction (McFadden, 2013 (75)) Frequencies inducing coherent states of brain activities to act as a catalysator for information transfer between neuronal structures, electrical fields, and consciousness.

This idea of an Information Field that interacts through quantum processes with the matter has been substantiated by the models and research of interdisciplinary scientists such as:

- Einstein’s protégé David Bohm (76) (Theory of Hidden Variables)

- The Bohm Aharonov effect (77)

- Works of Stanford professor William Tiller (Tiller, 1993 (78)),

- Experiments of Roger Nelson (Nelson, Radin 2019 (79)),

- Evolution biologist William B. Miller (Miller, Torday, Baluska (80)),

- Russian cosmologist Nikolai Kozyrev (81), and

- German physicist and Heisenberg-student Burkhard Heim (82).

Guidance from the Information Field to Support Biological Processes

The Information Field is considered, by scientists such as Rupert Sheldrake (83), William Milleror
William Tiller, to be a guide to understanding the evolution of life on this planet ${ }^{9}$. Also, Robert Becker addresses the concept of the morphogenetic (shaping) field from which the blueprint of growth is derived. These electrical fields (according to Becker) contain all information about the development of life (Becker, et al. (84)).The development consists of nonlocal information accumulating as the memory of nature to develop higher-order and more complex systems. According to this model, each local change, development, and evolution is stored dynamically in the Information Field in order to non-locally guide random processes and to increase the complexity and coherence of life. The existence of such fields does not conflict with quantum theory and even suggests

\footnotetext{
9 "It is certain that information is being sent and received within and across the cell at all times, reverberates externally and has further reciprocal effects. The context of information transfer across a vast multicellular constituency is obviously complex and based upon receiver and sender characteristics, which is perforce a function of velocity that depends upon the medium of transfer and information type. Further yet, a great deal of it might be regarded as noise. The appropriate means of assessing its summation might be best considered as a complex Information Field, and in turn, such an active informational field has its epicenter within and overlaps every cell and projects beyond it. This is simply analogous to the more familiar concept of any cell having its own energy field that consists of its gradients and fringe effects. In the case of an Information Field, it is the summation of all the sources and receptors of information within the cell and extends outward into the external environment.
}

The term "field" is appropriate since there is no reason to suspect that there is any exclusivity for reception of information within any purported "network". Some players might be privileged based on field effects, e.g., amplitude or frequency, but it is likely an open system, more like a broadcast than a direct line.

Any concept of a Pervasive Information Field can be easily reconciled with self-awareness. It is an actualization in biologic terms of an informational set. Within this definition, it rationalizes the non-intuitive requirement of cellular boundaries towards purposive self-awareness. The cellular boundary delimits the informational field, shielding it from some distortions or deformations caused by external environmental variables and adjacent cellular field effects. The cell membrane creates the environment in which the integrity of the Information Field can be protected and coherently projected. Therefore, our typical biological frame of reference of material form can be redirected towards a larger concept of information space." (William Miller $2016(50))$ 
that random quantum processes can connect the communication between any dynamic system and the global non-local Information Field. In this model, the collapse of the wave function is not a purely random effect contradicting the designers of quantum physics such as Albert Einstein, Erwin Schrödinger, and De Broglie. Rather, it bridges the gap between the Unitarian development of the wave function and the seemingly meaningless collapse of the wave function. In the model of the Information Field, the random quantum processes like physical noise generators express the effects of the Information Field and of intention and consciousness (Henry Stapp 1993 (85)). The physical noise generator used in the proposed bioenergetic processes is based on open quantum processes in the $\mathrm{PN}$-junction (quantum shot noise) of a transistor or a diode $^{10}$ (Beenakker, Schönenberger, 2003 (86)).Even thermal components of a $\mathrm{PN}$-junction noise are ultimately caused by quantum events (Derek Abbott, 2013 (87)). The quantum nature of this process increases receptivity for quantum entanglements. In a paper published in Physics Today (Beenakker and Schönenberger 2003 (88)) the authors state that "The noise is the signal" was a saying used by Rolf Landauer, one of the founding fathers of mesoscopic physics. What he meant is that fluctuations in the time of a measurement can be a source of information that is not present in the time-averaged value. Therefore, "aphysicist may actually delight in noise”.

The principle of information pattern analysis in physical noise signals used in the proposed

\footnotetext{
${ }^{10}$ A p-n junction is a boundary or interface between two types of semiconductor materials, $p$-type and n-type, inside a single crystal of semiconductor. The "p" (positive) side contains an excess of holes, while the " $n$ " (negative) side contains an excess of electrons in the outer shells of the electrically neutral atoms there. This allows electrical current to pass through the junction only in one direction.
}

bioenergetic process for Information Field analysis is based on this assumption.

\section{Quantum Physics, Consciousness and Information}

Quantum mechanics includes both the knowledge of the observer and the observer's knowledge-acquiring actions. Thus, an observer's intentions can influence physical behavior, as Descartes suggested (Schwartz, Stapp, Beauregard 2004 (89)).Following the ideas of Werner Heisenberg, John von Neumann, Alfred North Whitehead, Eugene Wigner, and others, Stapp developed a theory of human consciousness as $a$ process(Henry Stapp (90)). Von Neumann and Wigner argued that the collapse of the wave function in any measurement process depends on the actions of a conscious observer (Wigner 1972 (91)) (Atmanspacher 2004 (92)).

Stapp's premise is similar to those of Roger Penrose, the Australian philosopher David Hodgson (93), and the British philosopher Michael Lockwood. Penrose and Hodgson consider "nonlocal" quantum effects over areas of the brain to be much larger than the microscopic size of single atomic processes.

All these researchers explore the implications of quantum mechanical nonlocality that were first made famous by the 1935 Einstein-Podolsky-Rosen EPR thought experiment (94). When he first presented his conclusions regarding quantum mechanics at the 1927 Solvay conference, Einstein had worried about nonlocality (faster-than-light information about probabilities) in the collapse of a single-particle wave function.

Stapp's unique contribution to the free will problem is his claim that the observer's free choice of which experiment to perform, combined with the Quantum Zeno Effect, allows the observer, 
by his own free choices, to hold stably in place a chosen brain activity that would normally fade away (Stapp 2012 (95)).

In his book Mind, Matter,and Quantum Mechanics, Henry Stapp (96) states on pages $271 \mathrm{f}$ :

"Still, I believe that there will be nearunanimous agreement among quantum physicists that, to the extent that a rationally coherent conception of physical reality is possible, this reality will be informational in character, not material. For the whole language of the quantum physicist, when he is dealing with the meaning of his symbols, is in terms of information, which an agent may or may not choose to acquire, and in terms of Yes-or-No answers that constitute bits of information.

"Just getting that one idea across could make a significant inroad into the corruptive materialist outlook that, more than threequarters of a century after its official demise as a basic truth about nature, still infects so many minds.”

Henry Stapp`s significant contribution to the discussion about the interpretation of quantum physics - which he arrived at in agreement with physicists such as Roger Penrose-is the conviction that material nature can be influenced by human consciousness through macroscopic quantum states in the brain (Hameroff, Penrose 2017 (97)), and that this level of reality is the level of information, which in relation to mind and consciousness is not just epistemic but of a fundamental ontological nature.

This is called the Information Field, through which the intention of consciousness can influence quantum processes like the physical noise generator contained in the proposed bioenergetic process. This was experimentally proven by the PEAR Lab experiments (Jahn, Dunne, 1999 (98)) and used as the scientific basis for Roger Nelsons Global Consciousness Project (Roger Nelson 2008 (99)). The concept of an ontological level of information (Information Field), influenced by consciousness and creative acts of the mind (Stapp 1993 (100)), and interacting to matter through the stochastic collapse of the wave function is a common conclusion of many researchers, including Henry Stapp (Penrose, Laszlo, von Neumann, David Bohm,etc), (Martin, Carminati 2017 (101); Penrose, Hameroff 2017 (102)). In this way, the concept of a field of information (or Information Field)has a solid scientific basis as the next step beyond the limited and naïve belief that physical nature is purely ruled by random processes and that consciousness is not a fundamental ontic reality that has no effect on matter. The very concept of a quantum theory and the experiments of PEAR Lab (Jahn, Dunne 1999 (103)) actually defeat this unsophisticated, materialistic version which continues to maintain the mechanistic worldview of Cartesian philosophy and Newtonian Physics.

Information Field controlled Frequency Applications

The discussed bioenergetic process uses the quantum-based physical noise generator to acquire guiding information from the global Information Field and the intention of the user (PEAR Lab 1999 (104)) (Jahn, Dunne 2005 (105)) ${ }^{11}$ (Jahn, Dunne

\footnotetext{
${ }^{11}$ Robert Jahn and Brenda Dunne after two decades of research, at the PEAR lab of Princeton University, on influence of intention on physical random generators have concluded that intention, which is a content or function of consciousness, can interact with physical systems like physical noise generators, because it is included in the model of quantum physics, which they propose, as a wave function, which for example includes the specific intention as its content and interacts with an environment represented in the theory as a physical potential field:

"The model proposed in (Jahn and Dunne 1986 (106)) takes the position that reality is constituted only in the interaction of consciousness with its environment, and therefore that any
} 
1986 (106)) to select a dynamic sequence of frequencies to guide the bioenergetic process towards:

- $\quad$ strong bioenergetic potential,

- balanced distribution of energetic levels of the matrix surrounding the cells, and

- the order/coherence of the whole bioenergetic organism (the body-related component of the individual Information Field).

The selective process of the Information Fieldcontrolled choice of frequencies gives the organism a clear direction towards bioenergetic and bioelectric coherence and balance. According to the WQT (Walach 2005 (107)), all the parts of the global system are quantum entangled and form a wholeness of quantum inseparability.

The global Information Field, the bioelectric field, the physical noise generator, and the selected sequence of frequencies are the four components of a global system. According to the underlying intention of the Weak Quantum Theory, its four components are pair-wise quantum entangled. The physical noise generator acts as the central hub of this quadrupole system, connecting the

- global Information Field,

- bioenergetic field, and

- selected frequencies

...in a row of a circular informational channel.

scheme of conceptual organization developed to represent that reality must reflect the processes of consciousness as well as those of its environment. In this spirit, the concepts and formalisms of elementary quantum mechanics are appropriated via suitable metaphors to represent the characteristics of consciousness interacting with its environment. More specifically, we propose that if a consciousness is represented by a quantum mechanical wave function, and its environment, including its own physical corpus, is represented by an appropriate potential profile, Schrödinger wave mechanics yields eigen functions and eigen values that can be associated with the cognitive and emotional experiences of that consciousness in that environment."
1. Quantum Entangled Components of the Global System

Analogous to Harald Walach's explanation of complementary medicine processes by the WQT, two generalized quantum entanglements occur here, which are coupled in such a way - by a classical process of information transfer- that quantum teleportation of states can occur throughout the process (Walach 2005 (108)).In this case, the physical noise generator (A) and the global Information Field (C) are entangled with the bioenergetic field (B) of the user (the letters A, B, C, and $\rho_{\mathrm{i}}$ are classically used in the context of quantum teleportation).

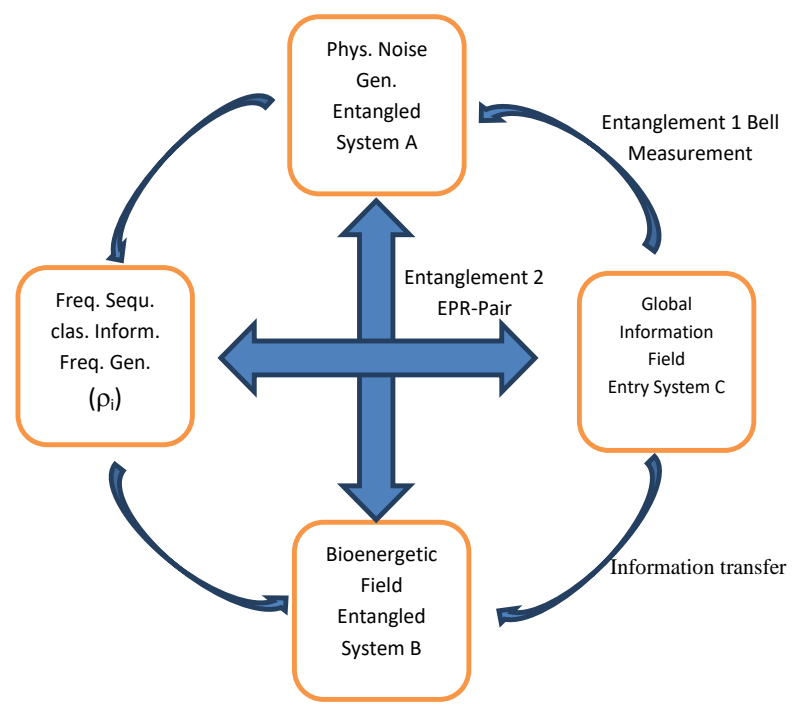

Figure 2. Double quantum entanglement in Information Field controlled frequency therapy in relation to quantum teleportation

These entanglements correspond to the mechanisms and results of the PEAR Lab research. Thus:

- the pattern of the global Information Field(C), as intended for the user,

- can be transferred to the bioenergetic field (B) of the user under one condition,

- namely that the information, how the global Information Field (C)

- has influenced the physical noise generator (A) 
- is transferred to the bioenergetic field of the user in the classical way i.e. via the selected frequencies $\left(\rho_{\mathrm{i}}\right)$.

In the context of the physical mechanism of quantum teleportation, the second entanglement between the bioenergetic field and the physical noise generator is called an EPR pair of the Einstein-Podolsky-Rosen paradox, (109) and the first entanglement is called Bell measurement.

The following section describes the basic relationships of quantum teleportation using a simple diagram.

\section{Double Entanglement and Quantum Teleportation}

In the following figure, quantum teleportation is shown in a simplified form with all essential elements (110). The basic transmission path consists of the two photons, A and B, which are entangled by a quantum process. In this case, the bioenergetic field (B) of the user and the physical noise generator (A) are quantum entangled in a generalized sense. This entanglement corresponds to an extended application of the PEAR effect (111).

If the photon $\mathrm{C}$ is now entangled with photon $\mathrm{A}$ by a Bell measurement, the quantum state of photon $C$ is transferred to photon $\mathrm{B}$, but this is only effectively accomplished when information $\left(\rho_{\mathrm{i}}\right)$ about the Bell measurement is transferred to photon $\mathrm{B}$ via a classical channel (112).

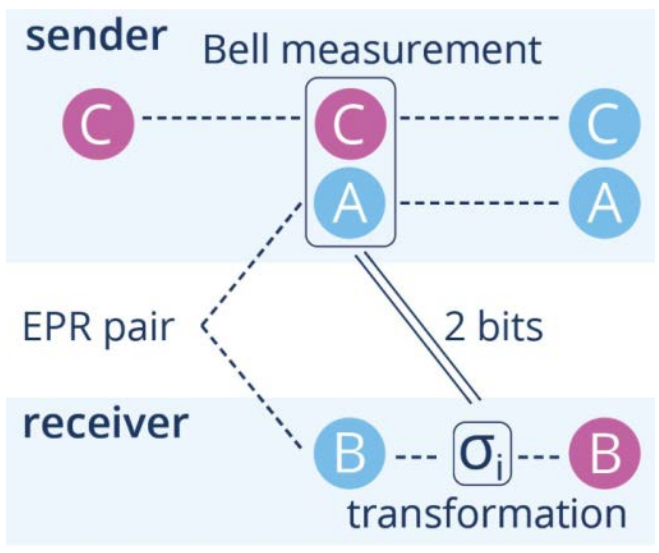

Figure 3. Principle of quantum teleportation based on double quantum entanglement

A Bell measurement does not provide information about the quantum state of the two photons $\mathrm{A}$ and $\mathrm{C}$, but provides information about the Bell state containing information about the global state of both photons as a whole quantum object. Here, parts of the information about the individual photons remain hidden. In this measurement, both photons are entangled. In order to recover the information of the original photon $\mathrm{C}$ during measurement of photon $\mathrm{B}$, the information about the Bell state of the A-C system must be transferred in a classical way $\left(\rho_{\mathrm{i}}\right)$ to decode photon B. In this example, it is done by applying the frequencies determined by the physical noise generator to the bioenergetic field of the user.

3. Double Quantum Entanglement in the Information Field Controlled Frequency Therapy The physical noise generator technically has the task of selecting frequencies that currently have the strongest resonance with the bioenergetic field of the user, according to the influence of the global Information Field at a given time. The physical noise generator (PNG)works for this purpose like the ones used in the experiments of Robert Jahn and Brenda Dunne in the PEAR Lab experiments (113). The extensive research of Roger Nelson on the use of PNGs in relation to the global field of consciousness 
also shows that noise can map field resonances of humans (114). The cumulative deviation of the values of the noise generator's signal from the expectation value is taken as a measure of the resonance of the respective frequency with the user's bioenergetic field. The correlation to the individual frequencies available for selection is established by loading them successively into the Ram memory of the process, whereby the entropy change of the physical noise is measured in each case. In generalized quantum teleportation, this measurement corresponds to the bell measurement between the input system $C$ (global Information Field) and the physical noise generator (A) in the sense of a PEAR correlation measurement and thus to the (generalized) entropy measurement. Likewise, there is a relation between the physical noise generator (A) and the user's bioenergetic field (B) analogous to the PEAR correlation, which is the second entanglement or EPR pair. Through this generalized entanglement, the noise becomes a representative of the user's field to create resonances to the global Information Field.

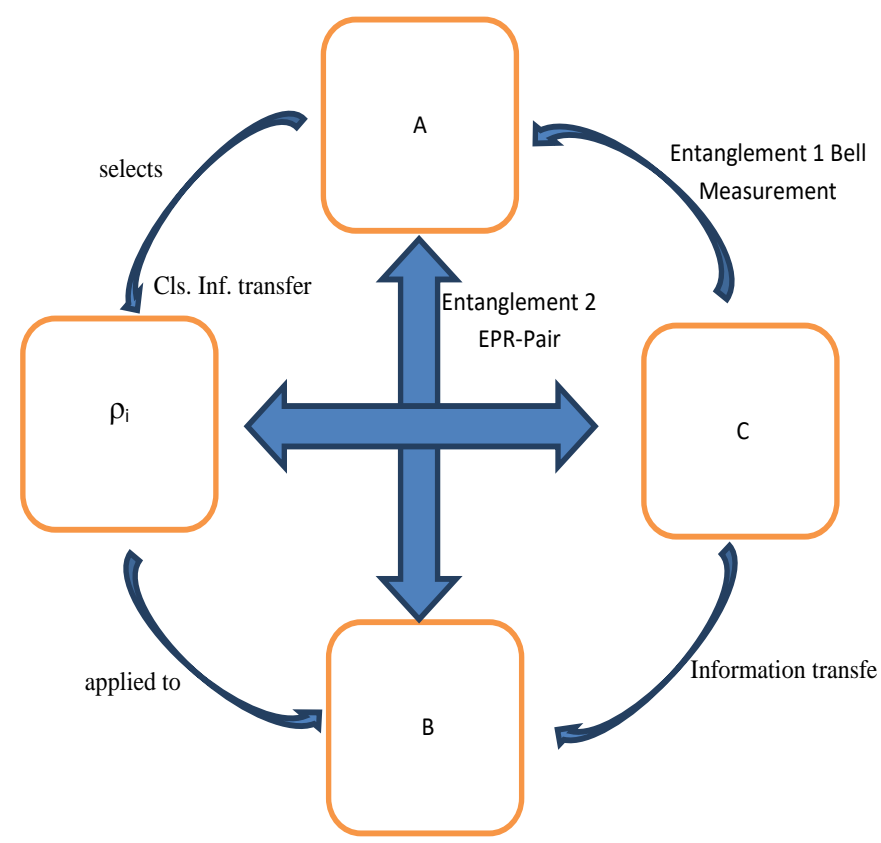

Figure 4. Principle of quantum teleportation in the quadrupole shape
4. Generalized Bell Measurement and Classical Information Transfer

This generalized Bell measurement takes place on the entangled system consisting of the global Information Field (C) and the noise generator (A). The connection between these two components is mediated by the PEAR effect (115). The noise generator determines a certain frequency sequence $\left(\rho_{\mathrm{i}}\right)$ from the multitude of possible frequency combinations. This sequence now contains the information about the unit of the system consisting of:

- the noise generator $(\mathrm{A})$ and

- the global Information Field (C),

- which is classically transferred locally to the bioenergetic field (B)

- of the user by the frequency generator $\left(\rho_{\mathrm{i}}\right)$.

Thus, the original information pattern of the global Information Field C becomes decodable for system B, i.e. it is available to the individual field for bioenergetic balancing. This specific quantum information of the global Information Field (C) is transferred to the bioenergetic field (B) and in this way is mediated by the frequency sequence $\left(\rho_{\mathrm{i}}\right)$.

\section{The Importance of Amplitude in Frequency Therapy} The influence of the amplitude of the applied frequencies on the described effect has not been researched in detail. Under certain circumstances, the reduction of the amplitude could be compared with an increase in potentiation (dilution) in homeopathy, so that-with low amplitude-a shift of the effect from the physiological to the quantum field occurs. If the frequencies are applied in the upper micro-current range between 500 and 1000 micro-amperes(e.g. globally via wrist electrodes), it is to be expected that the classical effect of microcurrent therapy will overlap with specific frequencies on the bioelectric milieu in the body and 
the effect based on double quantum entanglement described above. By adjusting the amplitude, it might then be possible to regulate between the two types of effect. This needs further research.

5. Reversing the Direction of Entanglement as an Alternative Mode

Frequency therapy controlled from the Information Field can also be derived in an alternate entanglement process by looking at the direction of the information flow in the opposite direction. This second variant isdescribed in thefollowingdiagram:

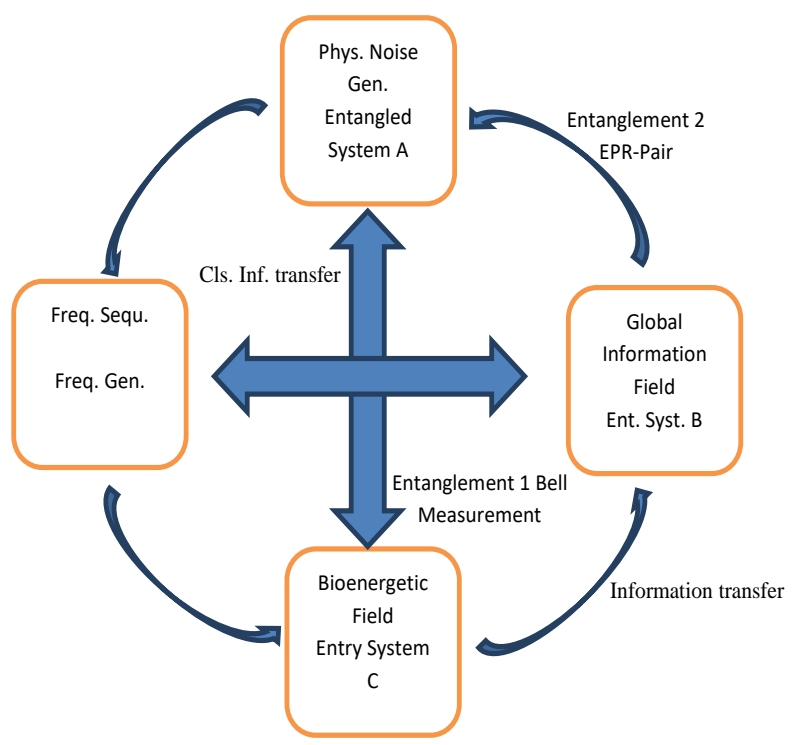

Figure 5. Variant two of the double entanglement process

In this mode, the original EPR pair corresponding to photons $\mathrm{A}$ and $\mathrm{B}$ consists of the physical noise generator (A) and the global Information Field (B). This entanglement is again mediated by the PEAR analog measurement. The second PEAR-like generalized entanglement between the bioenergetic field (C) and the physical noise generator (A) corresponds to the Bell measurement, whereby the pathology of the bioenergetic field is transferred to the overarching Bell state corresponding to the pathological network under consideration. The classical transmission path of the Bell state necessary for decoding the quantum state takes place from the physical noise generator (A) to the selected frequency sequence, which in turn is assigned to the information pattern of the global Information Field (B). In this way, the quantum state of the pathological network can now be transferred from the bioenergetic field (C) to the global Information Field (B). This transfer corresponds, as suggested by Harald Walach (116), to a suction of the pathological information (C) through the global Information Field (B). The no-cloning theorem of quantum theory states that the quantum state copied in this way must be deleted in the original system $\mathrm{C}$ of the bioenergetic field since it cannot exist twice.

Since both processes are possible at the same time, the resulting process is a superposition of both processes, so that-depending on the point of view - one of the two directions comes to the fore. Thus, both a transfer of harmonizing information from the global Information Field and absorption of pathological information from the bioenergetic field can take place.

Self-Treatment to improve the sense of Coherence and general Wellbeing using Information Field controlled Frequency Therapy

\section{Design}

The study was designed as a stratified open design trial of two weeks treatment duration with a measurement point at the beginning and at the end of the study.

A newly developed frequency-modulated microcurrent application was distributed to all experienced users of a bioenergetic device called Healy which works according to the described 
mechanisms of this paper. These persons were asked to participate in the study. All persons who consented to study participation were provided with the initial questionnaire. The participants were advised to use the new program twice per day (45 minutes duration each) and if possible, to refrain from using other Healy applications during the two weeks study period. All participants who completed the first questionnaire were provided with the final questionnaire after 14 days.

\section{Treatment Device / Treatment Application}

The Healy bioenergetic device has to be attached to the body via electrodes. The electrodes were exclusively placed as conductive wrist bands at the wrists so that the microcurrent would flow through the upper part of the body. Healy uses frequencies from $0.1 \mathrm{~Hz}$ to $1 \mathrm{MHz}$. Healy applies an electrical current between $200 \mu \mathrm{A}$ and $1000 \mu \mathrm{A}$. The maximum applied voltage is $10 \mathrm{~V}$.

The Healy Coherence application combines 214 frequencies of a universal frequency pattern discovered recently by Dutch scientists (117) into an Information Field controlled Healy program. The coherence program's objective is to support the harmony of the Bioenergetic Field (BEF) and the sense of coherence.

\section{Stratification}

Stratification was conducted according to the answers to the question of whether the new program was used during the previous two weeks or not as given in the second questionnaire. This is resulting in two groups: Coherence users and Non-users of Coherence.

\section{Outcome Measures}

The primary outcome was the WHO5-Wellbeing scale (118), a 5-item scale that is widely accepted to measure wellbeing as a generic scale. The items are summed up to yield a sum score ranging from 0 to 25 and standardized on a percentage scale from 0 to 100 (sum score multiplied by 4).

As secondary outcome was an individualized score, the Measure Your Own Medical Outcome Profile (MYMOP) Score (119). Individuals are free to define up to three different concerns that they want to improve. Present burdens are rated on a 10-point numerical rating scale.

Adverse events were elicited by an open question. The questionnaires were presented online after informed consent was received, and then again after two weeks.

Since the study was conducted in healthy, wellinformed volunteers giving informed consent, and no invasive measures are applied, ethical counsel was not sought and was not necessary according to local legal frameworks.

\section{Statistics}

Pre/post comparisons of the WHO-Q5-wellbeing scale and the MYMOP respectively are defined as primary and secondary outcome measures. Group comparisons (Coherence users versus Coherence Non-Users) were conducted by use of variance analytic methods. In case one single datapoint was missing, data were to be interpolated by the lastvalue-carried-forward method. If more than one single datapoint in a dataset was missing, the respective dataset was excluded from the analysis. 


\section{Results}

Three thousand five hundred and forty-five participants completed both questionnaires. 3164 (Coherence Group) participants of the study used the coherence program during the study at least once. 381 participants did not use the Coherence program (Non-Users group). Baseline data are presented in Table 1.

\begin{tabular}{cccc}
\hline & $\begin{array}{c}\text { Coherence } \\
(\mathrm{n}=3164)\end{array}$ & $\begin{array}{c}\text { No Coherence } \\
(\mathrm{n}=381)\end{array}$ & $\begin{array}{c}\text { Total } \\
(\mathrm{n}=3545)^{*}\end{array}$ \\
\hline Gender & & & \\
\hline Female & $2629(83 \%)$ & $305(80 \%)$ & $2934(83 \%)$ \\
\hline Male & $516(16 \%)$ & $74(19 \%)$ & $590(17 \%)$ \\
\hline DNS & $12(0,4 \%)$ & $1(0,2 \%)$ & $13(0.4 \%)$ \\
\hline Age Groups & & & \\
\hline $20-40$ & $298(9 \%)$ & $24(6 \%)$ & $322(9 \%)$ \\
\hline $40-60$ & $1823(57 \%)$ & $222(58 \%)$ & $2045(57 \%)$ \\
\hline $60-80$ & $1019(32 \%)$ & $133(34 \%)$ & $1152(32 \%)$ \\
\hline$>80$ & $24(1 \%)$ & $3(-\%)$ & $27(1 \%)$ \\
\hline
\end{tabular}

Table 1.Demographic Data per Group (Coherence User /Nonuser; absolute frequencies and percentages (per category and group); mean scores for WHO 5 and MYMOP scales, [95\% Confidence Intervals]. ${ }^{\star 3537 ~(8 ~ m i s s i n g s) . ~}$

\begin{tabular}{lll}
\hline & $\begin{array}{l}\text { Coherence } \\
(n=3164)\end{array}$ & $\begin{array}{l}\text { No Coherence } \\
(n=381)\end{array}$ \\
\hline WHO 5 & 52,9 & 55,3 \\
(0: worst; 100: best) & $\begin{array}{l}{[52,2 ; 53,7]} \\
n=3145\end{array}$ & $\begin{array}{l}{[53,1 ; 57,5]} \\
n=293\end{array}$ \\
\hline MYMOP1 & 7,33 & 7,17 \\
(0: best; 10: worst) & {$[7,26 ; 7,40]$} & {$[6,917,42]$} \\
& $n=3139$ & $n=276$ \\
\hline MYMOP2 & 6,99 & 6,82 \\
(0: best; 10: worst) & {$[6,93 ; 7,07]$} & {$[6,577,07]$} \\
& $n=3139$ & $n=275$ \\
\hline & 6,82 & 6,63 \\
MYMOP3 & {$[6,77 ; 6,91]$} & {$[6,376,89]$} \\
(0: best; 10: worst) & $n=3139$ & $n=267$ \\
& 20,51 & 20,24 \\
\hline
\end{tabular}

Table 2.Mean baseline scores for WHO 5 and MYMOP scales, [95\% Confidence Intervals]
The stratification yielded two quite comparable groups (see table 1). $83 \%$ of the participants were female. 58 percent or 2045 participants belonged to the middle-aged group between 40 and 60, and nearly one third were older than 60 years.

The baseline outcome data (table 2) were similarly well distributed. Data for the primary outcome, WHO5 were complete, whereas data for the MYMOP scales had some missing values. The testing procedure using t-test for paired samples yielded for the group using the coherence program at least once, a highly significant increase of the WHOQ5 scores by about 8.0 scoring points $(\mathrm{t}=-24.916$, $\mathrm{df}=3129, \mathrm{p}<0.00001$ ) (see figure 1 ). In the noncoherence group, the mean WHO-Score significantly decreases by about 6 scoring points in the course of the study $(\mathrm{t}=4,82$, $\mathrm{df}=203$, $\mathrm{p}$-value $<0.005$, see figure 1).An Anova with the factor use of Coherence (no/yes) indicates significant superiority of Coherence user versus non-users $(\mathrm{DF}=1, \mathrm{~F}=85,5$, $\mathrm{p}$ $<0,0001)$.

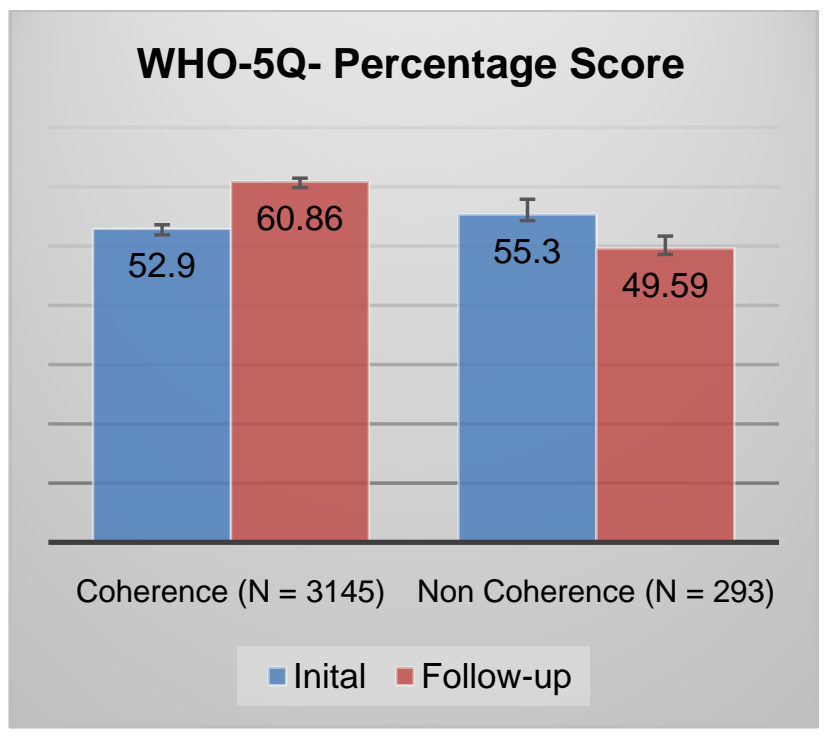

Figure 6. Linear Model of Main Outcome, pairwise group comparisons in general wellbeing score (WHO-5) at study start and study completion (error bars $=95 \%$ confidence interval). 


\begin{tabular}{|c|c|c|}
\hline \multicolumn{3}{|l|}{ Primary Outcome } \\
\hline & Coherence & No Coherence \\
\hline WHO5 Percent Sum & 60,86 & 49,59 \\
\hline Score & {$[60,14 ; 61,58]$} & {$[46,38 ; 52,80]$} \\
\hline \multicolumn{3}{|l|}{ Secondary Outcome } \\
\hline \multirow{3}{*}{ MYMOP1 } & 5,36 & 5,92 \\
\hline & {$[5,26 ; 5,44]$} & {$[5,60 ; 6,25]$} \\
\hline & $\mathrm{n}=3139$ & $n=278$ \\
\hline \multirow{3}{*}{ MYMOP2 } & 5,35 & 5,33 \\
\hline & {$[5,26 ; 5,43]$} & {$[5,02 ; 5,65]$} \\
\hline & $\mathrm{n}=3099$ & $n=269$ \\
\hline \multirow{3}{*}{ MYMOP3 } & 5,37 & 5,54 \\
\hline & {$[5,28 ; 5,45]$} & {$[5,24 ; 5,85]$} \\
\hline & $\mathrm{n}=3060$ & $n=268$ \\
\hline \multirow{3}{*}{ MYMOP Sum Score } & 15,88 & 16,43 \\
\hline & $15,65 ; 16,1037]$ & $15,58 ; 17,29]$ \\
\hline & $\mathrm{n}=3139$ & $n=278$ \\
\hline \multicolumn{3}{|c|}{ Issues during treatment phase (Adverse Events) } \\
\hline No & $2726(87 \%)$ & $270(76 \%)$ \\
\hline Yes & $396(13 \%)$ & $86(24 \%)$ \\
\hline Technical issues & 182 & 84 \\
\hline General questions & 25 & 2 \\
\hline Positive remarks & 108 & 0 \\
\hline $\begin{array}{l}\text { Health related issues } \\
\text { IAEs }\end{array}$ & 53 & 0 \\
\hline Not specified & 28 & 0 \\
\hline \multicolumn{3}{|c|}{ Adverse Event categories } \\
\hline Mood related & 19 & \\
\hline Pain related & 12 & \\
\hline Sleep related & 7 & \\
\hline Common cold & 3 & \\
\hline Other & 12 & \\
\hline
\end{tabular}

Table 3.Outcome Variables - Adjusted Mean Scores (Standard Errors, 95\% Confidence Intervals) of Main Outcome (WHO5 Postscore as a percentage) and Secondary Outcomes (MYMOP1-3 postscores, changes in summarized score) and Adverse Events (Frequency, Percent)

The secondary outcome, the three MYMOP scales, and the sum-score were tested using a paired t-test for pre/post value comparison in participants of the Coherence and participants of the Non-Coherence group separately. For both groups a highly significant decrease in summarized concern-load could be observed during the treatment by about 5,1 scoring points in the Coherence Group $(\mathrm{t}=40.592$, $\mathrm{df}=3043, \mathrm{p}$-value $\mathrm{p}<0.00001)$ respective 4,05 scoring points in the Non-Coherence Group $(\mathrm{t}=$ 7.6897, $\mathrm{df}=276$, $\mathrm{p}$-value $\mathrm{p}<0.00001$ ).

The decrease in symptom load is gradually higher in the Coherence group, but the group differences are not statistically significant $(\mathrm{t}=1.24$, $\mathrm{df}=317$, $\mathrm{p}$ value $=0,11$ for Mymop sum score).

55 participants (all in the Coherence group) reported health-related events. The most frequent complaints were mood-related (19), pain-related (12), sleeping difficulties (7), and seasonal infections (3). None of the reported issues has the characteristic of seriousness.

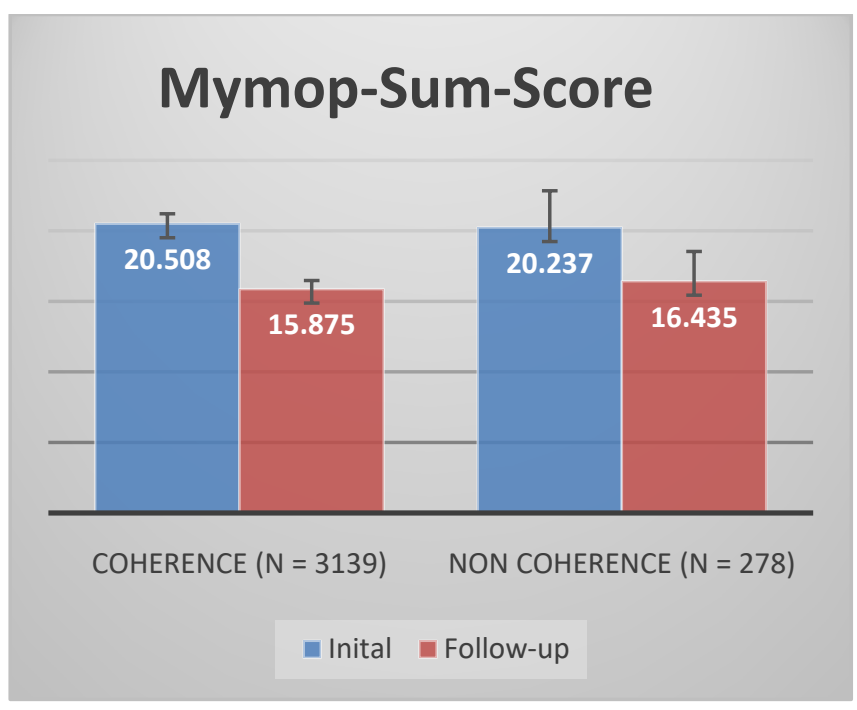

Figure 7. Secondary Outcomes MYMOP-Scores - sum-scores at study start and study completion (error bars $=95 \%$ confidence interval).

\section{Discussion}

We find clear evidence for the effectiveness of the new Coherence application, both WHO-wellness scores indicating improvement during the study. The effect is highly significant and clinically relevant (120). For the WHO-5 Item questionnaire measuring general wellbeing the changes during the study, phase have been beneficial for the Coherence group 
whereas the wellbeing index decreases in the untreated group.

Regarding the MYMOP scales, which measure individually chosen goals of improvement, the group differences are only gradually (decrease in symptom load in both groups) and not significant. For this item, it seems that awareness per se is the main trigger for a short-term decrease in concern-burden.

We conclude that frequency-modulated microcurrent applications using Healy are effective in improving general wellbeing in medically healthy volunteers. Because the mode of action of frequency-modulated microcurrent applications is not fully understood, and all data of this trial were analyzed in an exploratory sense, this should be investigated by additional more controlled trials.

\section{Acknowledgments}

We would like to thank Dr. Peter Marmann of Healy $\mathrm{GmbH}$ (Germany) for the statistical analysis and conducting of the study.

\section{Conflict of Interest}

Dr. Peter Marmann is employed as a senior specialist for clinical studies at Healy GmbH (Germany) and Marcus Schmieke is the inventor of the Healy device.

\section{References}

1. F A Popp, W Nagl, K H Li, W Scholz, O Weingärtner, R Wolf, Biophoton Emission. New Evidence for Coherence and DNA as Source, Cell Biophys. 1984 Mar;6(1):33-52.doi: 10.1007/BF02788579. https://doi.org/10.1007/BF02788579

2. F. A. Popp und Y. Yan: Delayed luminescence of biological systems in terms of coherent states. In: Physics Letters. Bd. A 293 (2002), S. 93-97. https://doi.org/10.1016/S0375-9601(01)00831-3

3. F. A. Popp, J. J. Chang, A. Herzog, Z. Yan und Y. Yan: Evidence of non-classical (squeezed) light in biological systems. In: Physics Letters. Bd. A $293 \quad$ (2002), $\quad$ S. 98-102. https://doi.org/10.1016/S0375-9601(01)00832-5

4. J I Jacobson, On the Electro-Magnetic Nature of Life, Panminerva Med. Oct-Dec 1989;31(4):151-65., PMID: 2633105

5. F A Popp, W Nagl, K H Li, W Scholz, O Weingärtner, R Wolf, Biophoton Emission. New Evidence for Coherence and DNA as Source, Cell Biophys. 1984 Mar;6(1):33-52. https://doi.org/10.1007/BF02788579

6. Francisco Di Biase, The unified Field of Consciousness, Unified Field Mechancis, A.R.L. Kaufmann, H. Louis, P. Rowlands, 2016). https://doi.org/10.1142/9789814719063 0025
7. K. J. Hintz, G.L. Yount, I. Kadar, G. Schwartz, R. Hammerschlag, S. Lin, BioEnergy Definitions and Research Guidelines, Published in Alternative Therapies in Health \& Medicine, Vol. 9, pp. 13-30 (2003). (January 29, 2003 Version 3.8)

8. Shyang Chang. The Meridian System and Mechanism of Acupuncture-A Comparative Review. Part 1: The Meridian System, Taiwan J Obstet Gynecol. 2012 Dec;51(4):506-14. https://doi.org/10.1016/j.tjog.2012.09.004

9. Hui-Chu Chiang, Hen-Hong Chang, Po-Yu Huang, Mutsu Hsu, On the Qi Deficiency in Traditional Chinese Medicine, Taiwan J ObstetGynecol. $2014 \quad$ Sep;53(3):317-23 https://doi.org/10.1016/j.tjog.2013.06.013

10. Primas, H (2003), Time-Entanglement Between Mind and Matter, Mind and Matter Vol. 1, 81-119.

11. Lucadou, W., Römer, H. \&Walach, H., Synchronistic phenomena as entanglement correlation in generalized quantum theory. [Synchronistic Phenomena as Entanglement Correlations in Generalized Quantum Theory]. In Journal for Parapsychology and Frontier Areas of Psychology, Editors: Bauer (January 2007); German: Synchronistische Phänomeneals Verschränkungskorrelation in der Verallgemeinerten Quanten theorie. [Synchronistic Phenomenaas Entanglement Correlations in Generalized Quantum Theory]. von Lucadou, W., Römer, H. \&Walach, H. In Zeitschrift für Parapsychologie und Grenzgebiete der Psychologie, Editors: Bauer (Januar 2007).

12. Atmanspacher $\mathrm{H}$, Römer $\mathrm{H}$, Walach $\mathrm{H}$. Weak quantum theory: Complementarity and entanglement in physics and beyond. Foundations of Physics 2002;3:379406.https://doi.org/10.1023/A:1014809312397

13. Anton Zeilinger, Fundamentals of quantum information, Published 1 March 1998 - Physics World, Volume 11, Number 3. https://doi.org/10.1088/2058-7058/11/3/29

14. Harald Walach, Generalized Entanglement: A New Theoretical Model for Understanding the Effects of Complementary and Alternative Medicine, J Altern Complement Med. 2005 Jun;11(3):549-59. https://doi.org/10.1089/acm.2005.11.549

15. Aharonov and D. Bohm. Significance of Electromagnetic Potentials in the Quantum Theory. Y. Phys. Rev. 115, 485 $\begin{array}{llll}\text { Published } & 1 & \text { August }\end{array}$ https://doi.org/10.1103/PhysRev.115.485

16. D. Bouwmeester, J. W. Pan, K. Mattle, M. Eibl, H. Weinfurter, A. Zeilinger. Experimental Quantum Teleportation, Nature 390, 575579 (1997). https://doi.org/10.1038/37539

17. Atmanspacher $\mathrm{H}$, Römer $\mathrm{H}$, Walach $\mathrm{H}$. Weak quantum theory: Complementarity and entanglement in physics and beyond. Foundations of Physics 2002;3:379-406 https://doi.org/10.1023/A:1014809312397

18. William A. Tiller, What are subtle energies?, Journal of Scientific Exploration, Vol. 7, No. 3, 1993.

19. Rupert Sheldrake, Morphic Resonance, World Futures, The Journal of New Paradigm Research, (2006), 62(1-2), 31-41. https://doi.org/10.1080/02604020500406248

20. Ervin Laszlo, Science and the Akashic Field, (2004), Science and the Akashic Field: An integral theory of everything, Rochester, VT: Inner Traditions International.

21. William B. Miller Jr., Biological information systems: Evolution as cognition-based information management, Progress in Biophysics and Molecular Biology, Volume 134, May 2018, Pages 1-26. https://doi.org/10.1016/j.pbiomolbio.2017.11.005

22. William B. Miller, Jr., Cognition, Information Fields and Hologenomic Entanglement: Evolution in Light and Shadow,Biology (Basel). 2016 Jun; 5(2): 21., US National Library 
of Medicine, National Institutes of Health. https://doi.org/10.3390/biology5020021

23. Lloyd S. Computational capacity of the universe. Phys Rev Lett. 2002 Jun 10;88(23):237901. https://doi.org/10.1103/PhysRevLett.88.237901

24. Unger, Roberto Mangabeira; Smolin, Lee (2015). The Singular Universe and the Reality of Time: A Proposal in Natural Philosophy Hardcover - December 8, 2014. https://doi.org/10.1017/CBO9781139696487

25. E. Schrödinger, What Is Life? Cambridge University Press, Cambridge, 1944

26. A.S. Davydov, Biology and Quantum Mechanics, Pergamon Press, New York, 1982

27. Graham R. Fleminga, Gregory D. Scholes, Yuan-Chung Cheng, Quantum effects in biology, Procedia Chemistry 3 (2011) 3857 ,22nd Solvay Conference on Chemistry. https://doi.org/10.1016/j.proche.2011.08.011

28. ThiloHinterberger, Nikolaus von Stillfried, The Concept of Complementarity and its Role in Quantum Entanglement and Generalized Entanglement, in Axiomathes 23(3) • September 2012. https://doi.org/10.1007/s10516-012-9187-y

29. Atmanspacher $\mathrm{H}$, Römer $\mathrm{H}$, Walach $\mathrm{H}$. Weak quantum theory: Complementarity and entanglement in physics and beyond. Foundations of Physics 2002;3:379-406. https://doi.org/10.1023/A:1014809312397

30. Harald Walach, Generalized Entanglement: A New Theoretical Model for Understanding the Effects of Complementary and Alternative Medicine, J Altern Complement Med. 2005 Jun;11(3):549-59. doi: 10.1089/acm.2005.11.549. https://doi.org/10.1089/acm.2005.11.549

31. Schmieke, M. (2021). Bohm`s Quantum Potential Approach to Consciousness from the Perspective of a Four-Valued Logic. Dev Sanskriti Interdisciplinary International Journal, 17, 01-12. https://doi.org/10.36018/dsiij.v17i.206

32. Stuart Hameroff, Roger Penrose, Orchestrated reduction of quantum coherence in brain microtubules: A model for consciousness,Mathematics and Computers in Simulation 40 (1996) 453-480. https://doi.org/10.1016/0378-4754(96)80476-9

33. Carlo Dal Lin, Anna Poretto, Marta Scodro, Martina Perazzolo Marra, Sabino Iliceto, Francesco Tona, Coronary microvascular and endothelial function regulation: Crossroads of psychoneuroendocrineimmunitary signals and quantum physics [Part B], Department of Cardiac, Thoracic and Vascular Sciences, Padua University School of Medicine, Via Giustiniani 2, 35100 Padua, Italy, 2015. https://doi.org/10.15761/JIC.1000136

34. Francis Martin, Federico Carminati, Giuliana Galli Carminati, Synchronicity, Quantum Information and the Psyche, in Quantum Physics of Consciousness 2017

35. Lee Smolin, Temporal relationalism, Perimeter Institute for Theoretical Physics, Physics; arXiv: History and Philosophy of Physics. June 1, 2018

36. Atmanspacher $\mathrm{H}$, Römer $\mathrm{H}$, Walach $\mathrm{H}$. Weak quantum theory: Complementarity and entanglement in physics and beyond. $\begin{array}{llll}\text { Foundations of 2002;3:379-406. } & \end{array}$ https://doi.org/10.1023/A:1014809312397

37. Larissa Brizhik, Enrico Chiappini, PatriziaStefanini, GiuseppeVitiello, Modeling Meridians Within the Quantum Field Theory, Journal of Acupuncture and Meridian Studies, Volume 12, Issue 1, February 2019, Pages 29-36 https://doi.org/10.1016/j.jams.2018.06.009

38. Mei-na Yang, Jin-xiang Han, Review and analysis on the meridian research of China over the past sixty years, Chinese Journal of Integrative Medicinevolume 21, pages394-400(2015) https://doi.org/10.1007/s11655-015-2168-4
39. R P Bajpai, Quantum Coherence of Biophotons and Living Systems, Indian J Exp Biol. 2003 May;41(5):514-27. PMID: 15244274

40. Marco Bischof, Biophotons, The light in our cells, Zweitausendeins, 8. Auflage 1996

41. Ahsan H Khandoker, Chandan K Karmakar, Marimuthu Palaniswami, Analysis of Coherence Between Sleep EEG and ECG Signals During and After Obstructive Sleep Apnea Events Conf Proc IEEE Eng Med Biol Soc. 2008;2008:3876-9. https://doi.org/10.1109/CIC.2008.4749134

42. Michael von Brück, Weizsäcker and Indian Philosophy - A New Beginning in Negotiating the Dualism of Mind and Matter? Acta HistLeopoldina. 2014;(63):539-60.

43. Weizsäcker, Carl Friedirch von; Krishna, Gopi, Yoga and the Evolution of Consciousness - The Scientific Basis of Spiritual Experience, Weizsäcker, Crotona Verlag GmbH, 2010, p. 57 (german)

44. Primas, H (2003), Time-Entanglement Between Mind and Matter, Mind and Matter Vol. 1, 81-119

45. Maicol A. Ochoa,Wolfgang Belzig, Abraham Nitzan, Simultaneous weak measurement of non-commuting observables: a generalized Arthurs-Kelly protocol, Scientific Reportsvolume 8, Article number: 15781 (2018). https://doi.org/10.1038/s41598-01833562-0

46. Atmanspacher $\mathrm{H}$, Römer $\mathrm{H}$, Walach $\mathrm{H}$. Weak quantum theory: Complementarity and entanglement in physics and beyond. Foundations of Physics 2002;3:379-406. https://doi.org/10.1023/A:1014809312397

47. Harald Walach, Generalized Entanglement: A New Theoretical Model for Understanding the Effects of Complementary and Alternative Medicine, J Altern Complement Med. 2005 Jun;11(3):549-59. https://doi.org/10.1089/acm.2005.11.549

48. F A Popp, W Nagl, K H Li, W Scholz, O Weingärtner, R Wolf, Biophoton Emission. New Evidence for Coherence and DNA as Source, Cell Biophys. 1984 Mar;6(1):33-52.doi: 10.1007/BF02788579. https://doi.org/10.1007/BF02788579

49. Thomas Görnitz, Verlag der Carl Friedrich von Weizsäcker Stiftung, 2012, p. 132

50. Mannheimer, Jeffrey S., 2005The Effect of Microcurrent Stimulation on ATPSynthesis in the Human Masseter as Evidenced by31P Magnetic Resonance Spectroscopy, Seton Hall University, Dissertation.

51. William B. Miller, Jr., Cognition, Information Fields and Hologenomic Entanglement: Evolution in Light and Shadow,Biology (Basel). 2016 Jun; 5(2): 21., US National Library of Medicine, National Institutes of Health, p.13 https://doi.org/10.3390/biology5020021

52. William B. Miller, Jr., Cognition, Information Fields and Hologenomic Entanglement: Evolution in Light and Shadow,Biology (Basel). 2016 Jun; 5(2): 21., US National Library of Medicine. National Institutes of Health, p.26 https://doi.org/10.3390/biology5020021

53. William B. Miller, Jr., Cognition, Information Fields and Hologenomic Entanglement: Evolution in Light and Shadow,Biology (Basel). 2016 Jun; 5(2): 21., US National Library of Medicine. National Institutes of Health, p.9. https://doi.org/10.3390/biology5020021

54. Geesink, J.H. and Meijer, D.K.F. (2017) Electromagnetic Frequency Patterns that Are Crucial for Health and Disease Reveal a Generalized Biophysical Principle: The GM Scale. Quantum Biosystems, 8, 1-16.

55. Geesink,JH,Meijer, DKF. Quantum Wave Information of Life Revealed: An Algorithm for Electromagnetic Frequencies that Create Stability of Biological Order, with Implications for Brain 
Function and Consciousness NeuroQuantology 2016a; 1: 106-125. https://doi.org/10.14704/nq.2016.14.1.911

56. Geesink, J H, Jerman, I, Meijer, D K F. Water: the cradle of life in action, cellular architecture is guided by coherent quantum frequencies as revealed in pure water, Water Journal, 2020.

57. Geesink J H, Jerman I, Meijer D K F. Clay minerals: information network linking quantum coherence and first life, 2020.

58. Meijer D K F, JermanI ,Melkikh AV and Sbitnev V I , (2020a). Consciousness in the Universe is Tuned by a Musical Master Code. Part 1: A Conformal Mental Attribute of Reality : Quantum Biosystems | 2020 | Vol 11 | Issue 1 | Page 72-107.

59. Geesink, J H, Jerman, I, Meijer, D K F. Water: the cradle of life in action, cellular architecture is guided by coherent quantum frequencies as revealed in pure water, Water Journal, 2020.

60. Fröhlich H, 1968. Long-range coherence and energy storage in biological systems. Int. J. Quantum Chem. 2: 641-649. https://doi.org/10.1002/qua.560020505

61. Marco Bischof; Emilio Del Giudice, Communication and the Emergence of Collective Behavior in Living Organisms: A Quantum Approach; Molecular Biology International, v2013 n5 (2013): 1-19. https://doi.org/10.1155/2013/987549

62. Meijer, Geesink, Mathematical Structure for Electromagnetic Frequencies that May Reflect Pilot Waves of Bohm's Implicate Order, January 2018, Journal of Modern Physics 09(05):851-897. https://doi.org/10.4236/jmp.2018.95055

63. Meijer, Geesink, Mathematical Structure for Electromagnetic Frequencies that May Reflect Pilot Waves of Bohm's Implicate Order, January 2018, Journal of Modern Physics 09(05):851-897. https://doi.org/10.4236/jmp.2018.95055

64. Robert G. Jahn, Brenda J. Dunne.Two decades of PEAR: an anthology of selected publications; Princeton, NJ: School of Engineering and Applied Science, Princeton University, 1999.

65. Robert G. Jahn, Brenda J. Dunne. The PEAR Proposition, Journal of Scientific Exploration, Vol. 19, No. 2, pp. 195-245, 2005.

66. Radin, D. I. and Nelson R. D. (1989). "Evidence for consciousness-related anomalies in random physical systems." Foundations of Physics, Vol. 19, No. 12 https://doi.org/10.1007/BF00732509

67. Richard A. Blasband, The Ordering of Random Events by Emotional Expression,Journal of Scientific Exploration, Vol. 14, No. 2, pp. 195-216, 2000.

68. Nelson, Roger, Emotions, The Emotional Nature of Global Consciousness, Paper for the Bial Foundation 7th Symposium, March 2008, Global Consciousness Project. http://noosphere.princeton.edu

69. Roger D. Nelson, Dean Radin, Connected: The Emergence of Global Consciousness, ICRL Press 2019.

70. Schmidt, Helmut, and Henry Stapp. "PK with prerecorded random events and the effects of preobservation." The Journal of Parapsychology, vol. 57, no. 4, 1993, p. 331+. Gale Academic OneFile, Accessed 26 May 2020.

71. a) Schmidt, Helmut; Quantum Processes Predicted? New Scientist, October 1969, Seiten 114-115;.b) Schmidt, Helmut;Mental Influence on Random Events. New Scientist and Science Journal 1971, p. 757-768.; c).Schmidt, Helmut; PK Effecton Pre-Recorded Targets. The Journal for the American Society for Psychical Research 70 (1976) p. 267-291; d).Schmidt, Helmut; Can an Effect Precede Its Cause? A Model of a Non-causal World. Foundations of Physics 8, June 1978, p. 463-480. https://doi.org/10.1007/BF00708576; e).Schmidt, Helmut; Experiments in ESP and psychokinesis with the ATARI computer. San Antonio, Tex., 1982; f).Schmidt, Helmut; Randomness and the Mind. Looking for Psychic Effect in Games of Chance. Creative
Computing, April 1983, p. 180-186; g).Schmidt, Helmut; Additional effect for PK on pre-recorded targets", Journal of Parapsychology, 1985, Vol. 49, p 229-244; h) Schmidt, Helmut; Observation of a Psychokinetic Effect Under Highly Controlled Conditions. Mind Science Foundation 1993(also in Journal of Parapsychology 57 (1993), p. 351-372); i).Schmidt, Helmut; Random generators and living systems as targets in retro-PK experiments. Journal of the American Society for Psychical Research 91 (1997) p.1-13.

72. Frohlich, F. \& McCormick, D.A. 2010. Endogenous electric fields may guide neocortical network activity. Neuron, 67, (1) 129-143 available from:

PM:20624597.

https://doi.org/10.1016/j.neuron.2010.06.005

73. Singer, W. 2011. Dynamic formation of functional networks by synchronization. Neuron, 69, (2) 191-193 available from: PM:21262459. https://doi.org/10.1016/j.neuron.2011.01.008

74. McFadden, J. 2002a. Synchronous firing and its influence on the brain's electromagnetic field: evidence for an electromagnetic theory of consciousness. Journal of Consciousness Studies, 9, (4) 23-50.

75. McFadden, J, The CEMI Field Theory: Closing the loop, Journal of Consciousness Studies, Volume 20, Numbers 1-2, 2013, pp. 153-168(16).

76. David Bohm, A Suggested Interpretation of the Quantum Theory in Terms of "Hidden" Variables. . Phys. Rev. 85, 166 - Published 15 January 1952.https://doi.org/10.1103/PhysRev.85.166

77. Aharonov and D. Bohm. Significance of Electromagnetic Potentials in the Quantum Theory. Y. Phys. Rev. 115, 485 $\begin{array}{llll}\text { Published } & 1 & \text { August } & 1959 .\end{array}$ https://doi.org/10.1103/PhysRev.115.485

78. William A. Tiller, What are subtle energies?, Journal of Scientific Exploration, Vol. 7, No. 3, 1993.

79. Roger D. Nelson, Dean Radin , Connected: The Emergence of Global Consciousness, ICRL Press 2019.

80. William B. Miller Jr., John S. Torday, Frantisek Baluska, The NSpace episenome unifies cellular information space-time within Cognition-Based Evolution, Progress in Biophysics and Molecular Biology, 2019.

81. N.A.Kozyrev, Sources of Stellar Energy and the Theory of the Internal Constitution of Stars, In: Progress in Physics, 2005, v.3, 61-99.

82. Illobrand Von Ludwiger, Heim's Theory of Elementary Particle Structures, Journal of Scientific Exploration. Vol. 6, No. 3, pp. 217-231, 1992 0892-33 10192 O 1992 Society for Scientific Exploration

83. Rupert Sheldrake, Morphic Resonance, World Futures, The Journal of New Paradigm Research, (2006), 62(1-2), 31-41. https://doi.org/10.1080/02604020500406248

84. R O Becker, C H Bachman, Bioelectric Effects in Tissue, Clin OrthopRelat Res. Nov-Dec 1965;43:251-3. https://doi.org/10.1097/00003086-196500430-00024

85. H. P. Stapp., Mind, Matter and Quantum Mechanics, Springer, Berlin 1993. https://doi.org/10.1007/978-3-662-08765-7

86. Carlo Beenakker, Christian Schönenberger, Quantum Shot Noise, Fluctuations in the flow of electrons signal the transition from particle to wave behavior, Published in revised form in Physics Today, May 2003, page 37. https://doi.org/10.1063/1.1583532

87. Derek Abbott, To what extent is thermal noise a result of the quantum world? Discussion on researchgate, 2013.

88. Carlo Beenakker, Christian Schönenberger, Quantum Shot Noise, Fluctuations in the flow of electrons signal the transition from particle to wave behavior, Published in revised form in Physics Today, May 2003, page 37. https://doi.org/10.1063/1.1583532 
89. Jeffrey M. Schwartz, Henry P. Stapp and Mario Beauregard, Quantum physics in neuroscience and psychology: a neurophysical model of mind-brain interaction, 2004, Phil. Trans. R. Soc. B doi:10.1098/rstb.2004.1598. https://doi.org/10.1098/rstb.2004.1598

90. a) Stapp, Henry. (2011). Nerve Terminals and the Need to Use Quantum Theory. 10.1007/978-3-642-18076-7_4. https://doi.org/10.1007/978-3-642-18076-7_4; b)Stapp, Henry, Reply to a Critic: "Mind Efforts, Quantum Zeno Effect and Environmental Decoherence", 2012/12/15, NeuroQuantology. https://doi.org/10.14704/nq.2012.10.4.619; c)Stapp, Henry. (2011). Application to Neuropsychology. https://doi.org/10.1007/978-3642-18076-7_8; d). Stapp, Henry. (2011). The Physical Effectiveness of Conscious Will and the Quantum Zeno Effect. https://doi.org/10.1007/978-3-642-18076-7_6; e)Stapp, Henry. (2011). Actions, Knowledge, and Information. https://doi.org/10.1007/978-3-642-18076-7_3; f). Schwartz, Jeffrey \& Stapp, Henry \& Beauregard, Mario \& Plaza, Westwood. (2004). Quantum Physics In Neuroscience And Psychology: A New Model With Respect To Mind/Brain Interaction; g) Stapp, H; Schwartz, J. M; Beauregard, M. (2005). Quantum theory in neuroscience and psychology: A neurophysical model of mind-brain interaction. Philosophical Transactions of the Royal Society of London, Series B. 360 (1458): 1309-1327. https://doi.org/10.1098/rstb.2004.1598

91. Eugene P. Wigner, The Place of Consciousness in Modern Physics,Philosophical Reflections and Syntheses pp 261-267, 1972. https://doi.org/10.1007/978-3-642-78374-6 21

92. Harald Atmanspacher, Quantum theory and consciousness: an overview with selected examples, Discrete Dynamics in Nature and Society, In memory of Ilya Prigogine, Volume 2004, https://doi.org/10.1155/S102602260440106X

93. David Hodgson, Quantum Physics, Consciousness, and Free Will, The Oxford Handbook of Free Will: Second Edition (2 ed.), 2011. https://doi.org/10.1093/oxfordhb/9780195399691.003.0003

94. A. Einstein, B. Podolsky, N. Rosen: Can quantum-mechanical description of physical reality be considered complete? Phys. Rev. 47 (1935), S. 777-780. https://doi.org/10.1103/PhysRev.47.777

95. Stapp, Henry, Reply to a Critic: "Mind Efforts, Quantum Zeno Effect and Environmental Decoherence", 2012/12/15, NeuroQuantology. https://doi.org/10.14704/nq.2012.10.4.619

96. H. P. Stapp., Mind, Matter and Quantum Mechanics, Springer, Berlin 1993, p.107-108. https://doi.org/10.1007/978-3-662-08765$\underline{7}$

97. Penrose, Roger; Hameroff, Stuart; Consciousness in the Universe; Neuroscience, Quantum Space-Time Geometry and Orch Or Theory; 2017.

98. Robert G. Jahn, Brenda J. Dunne. Two decades of PEAR: an anthology of selected publications; Princeton, NJ: School of Engineering and Applied Science, Princeton University, 1999.

99. Nelson, Roger, Emotions, The Emotional Nature of Global Consciousness, Paper for the Bial Foundation 7th Symposium, March 2008, Global Consciousness Project. http://noosphere.princeton.edu.

100. H. P. Stapp., Mind, Matter and Quantum Mechanics, Springer, Berlin 1993. https://doi.org/10.1007/978-3-662-08765-7

101. Francis Martin, Federico Carminati, Giuliana Galli Carminati, Synchronicity, Quantum Information and the Psyche, in Quantum Physics of Consciousness 2017.

102. Penrose, Roger; Hameroff, Stuart; Consciousness in the Universe; Neuroscience, Quantum Space-Time Geometry and Orch Or Theory; 2017.

103. Jahn, Robert; Dunne, Brenda, Two decades of Pear, An ontology of selected publications, Princeton University, 1999.

104. Jahn, Robert; Dunne, Brenda, Two decades of Pear, An ontology of selected publications, Princeton University, 1999.
105. Jahn, R. G., \& Dunne, B. J. (1986). On the quantum mechanics of consciousness, with applicationto anomalous phenomena.Foundations of Physics, 16,721-772. https://doi.org/10.1007/BF00735378

106. Jahn, R. G., \& Dunne, B. J. (1986). On the quantum mechanics of consciousness, with applicationto anomalous phenomena.Foundations of Physics, 16,721-772. https://doi.org/10.1007/BF00735378

107. Harald Walach, Generalized Entanglement: A New Theoretical Model for Understanding the Effects of Complementary and Alternative Medicine, J Altern Complement Med. 2005 Jun;11(3):549-59. https://doi.org/10.1089/acm.2005.11.549

108. Harald Walach, Generalized Entanglement: A New Theoretical Model for Understanding the Effects of Complementary and Alternative Medicine, J Altern Complement Med. 2005 Jun;11(3):549-59. https://doi.org/10.1089/acm.2005.11.549

109. A. Einstein, B. Podolsky, N. Rosen: Can quantum-mechanical description of physical reality be considered complete? Phys. Rev. 47 (1935), S. 777-780. https://doi.org/10.1103/PhysRev.47.777

110. Nielsen, Michael (2010). Quantum Computation and Quantum Information. Cambridge University Press. https://doi.org/10.1017/CBO9780511976667

111. Robert G. Jahn, Brenda J. Dunne, The PEAR Proposition, Journal of Scientific Exploration, Vol. 19, No. 2, pp. 195-245, 2005.

112. Sych, Denis (7 January 2009). "A Complete Basis of Generalized Bell States". New Journal of Physics - via IOP Science. https://doi.org/10.1088/1367-2630/11/1/013006

113. Jahn, R. G., \& Dunne, B. J. (1986). On the quantum mechanics of consciousness, with applicationto anomalous phenomena.Foundations of Physics, 16,721-772. https://doi.org/10.1007/BF00735378

114. Nelson, Roger, Emotions, The Emotional Nature of Global Consciousness, Paper for the Bial Foundation 7th Symposium, March 2008, Global Consciousness Project.

115. Robert G. Jahn, Brenda J. Dunne. Two decades of PEAR: an anthology of selected publications; Princeton, NJ: School of Engineering and Applied Science, Princeton University, 1999.

116. Harald Walach, Generalized Entanglement: A New Theoretical Model for Understanding the Effects of Complementary and Alternative Medicine, J Altern Complement Med. 2005 Jun;11(3):549-59. doi: 10.1089/acm.2005.11.549. https://doi.org/10.1089/acm.2005.11.549

117. Geesink, J.H. and Meijer, D.K.F. (2017) Electromagnetic Frequency Patterns that Are Crucial for Health and Disease Reveal a Generalized Biophysical Principle: The GM Scale. Quantum Biosystems, 8, 1-16.

118. Bech P, Olsen L, Kjoller M, Rasmussen N: Measuring well-being rather than the absence of distress symptoms: a comparison of the SF-36 Mental Health subscale and the WHO-Five Well-Being Scale. Int J Methods Psychiatr Res 2003;12:85-91. https://doi.org/10.1002/mpr.145

119. Hermann K, Kraus K, Herrmann K, Joos S. A brief patientreported outcome instrument for primary care:German translation and validation of the Measure Yourself Medical Outcome Profile (MYMOP). Health Qual Life Outcomes 2014, 12: 112 https://doi.org/10.1186/s12955-014-0112-5

120. Bech P, Lunde M, Bech-Andersen G, Lindberg L, Martiny K: Psychiatric outcome studies (POS): does treatment help the patients? A Popperian approach to research in clinical psychiatry. Nord J Psychiatry 2007;61(suppl 46):4-34. https://doi.org/10.1080/08039480601151238 